\title{
How Sufficient Conditions are Related for Topology-Preserving Reductions
}

\author{
Kálmán Palágyi ${ }^{a}$
}

\begin{abstract}
A crucial issue in digital topology is to ensure topology preservation for reductions acting on binary pictures (i.e., operators that never change a white point to black one). Some sufficient conditions for topology-preserving reductions have been proposed for pictures on the three possible regular partitionings of the plane (i.e., the triangular, the square, and the hexagonal grids). In this paper, the relationships among these conditions are stated.
\end{abstract}

Keywords: digital topology, topology preservation, simple points, $P$-simple sets, hereditarily simple sets, general-simple deletion rules

\section{Introduction}

A binary picture on a grid is a mapping that assigns a color of black or white to each grid element called a point [15]. A regular partitioning of the 2D Euclidean space is formed by a tessellation of regular polygons (i.e., polygons having equal angles, and sides are all of the same length). There are exactly three polygons that can form such regular tessellations, these being the equilateral triangle, the square, and the regular hexagon [19] (see Figure 1). Although 2D digital pictures sampled on the square grid are generally assumed, triangular and hexagonal grids have also attracted significant interest $[4,15,19,20]$.
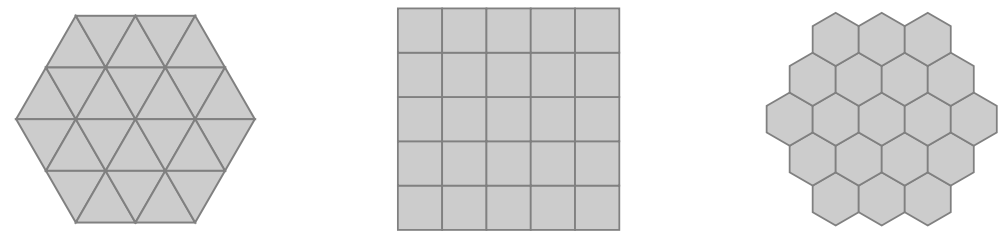

Figure 1: The three possible regular planar grids.

\footnotetext{
${ }^{a}$ Institute of Informatics, University of Szeged, Hungary, E-mail: palagyi@inf.u-szeged.hu
} 
A reduction transforms a binary picture only by changing some black points to white ones, which is referred to as deletion [15]. Reductions play a key role in some topological algorithms, e.g., thinning [5, 13, 15] and shrinking [6] algorithms.

Topology preservation is a major concern of reductions $[13,15]$. In this paper, five types of sufficient conditions for topology-preserving reductions acting on the three possible regular planar grids are presented, and the relationships among these conditions are revealed.

\section{Basic Notions and Results}

In this study, we apply the fundamental concepts of digital topology as reviewed by Kong and Rosenfeld [15]. Despite the fact that there are other approaches based on cellular/cubical complexes [16], here we shall consider the 'conventional paradigm' of digital topology.

\subsection{Binary Digital Pictures}

Let us denote the triangular, the square, and the hexagonal grids by $\mathcal{T}, \mathbb{Z}^{2}$, and $\mathcal{H}$, respectively, and throughout this article, if we will use the notation $\mathcal{V}$, we will mean that $\mathcal{V}$ belongs to $\left\{\mathcal{T}, \mathbb{Z}^{2}, \mathcal{H}\right\}$. The elements of the given grids (i.e., regular polygons) are called points. Two points are 1-adjacent if they share an edge and they are 2-adjacent if they share an edge or a vertex (see Fig. 2). Note that both relations are reflexive and symmetric. Now let us denote the set of points being $j$-adjacent to a point $p$ in the grid $\mathcal{V}$ by $N_{j}^{\mathcal{V}}(p)$, and let $N_{j}^{* \mathcal{V}}(p)=N_{j}^{\mathcal{V}}(p) \backslash\{p\}(j=$ $1,2)$. It is obvious that $N_{1}^{\mathcal{T}}(p) \subset N_{2}^{\mathcal{T}}(p), N_{1}^{\mathbb{Z}^{2}}(p) \subset N_{2}^{\mathbb{Z}^{2}}(p)$, and $N_{1}^{\mathcal{H}}(p)=N_{2}^{\mathcal{H}}(p)$.
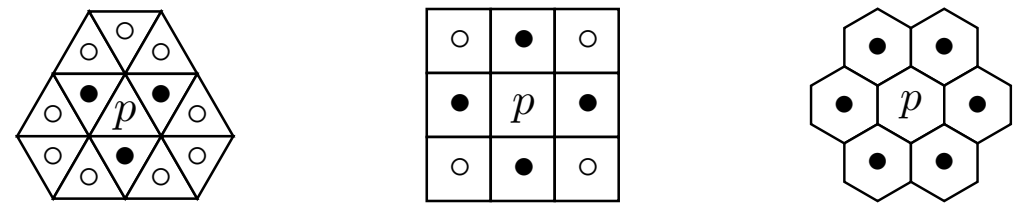

Figure 2: The adjacency relations studied on the three possible regular planar grids. Points that are 1-adjacent to the central point $p$ are marked ' $\bullet$ ', while points that are 2-adjacent but not 1 -adjacent to $p$ are denoted by ' $\circ$ '.

A sequence of distinct points $\left\langle p_{0}, p_{1}, \ldots, p_{m}\right\rangle$ is called a $j$-path from $p_{0}$ to $p_{m}$ in a non-empty set of points $X$ if each point of the sequence is in $X$ and $p_{i}$ is $j$-adjacent to $p_{i-1}$ for each $i=1,2, \ldots, m(j=1,2)$. Two points are said to be $j$-connected in a set $X$ if there is a $j$-path in $X$ between them. A set of points $X$ is $j$-connected in the set of points $Y \supseteq X$ if any two points in $X$ are $j$-connected in $Y$. A $j$-component of a set of points $X$ is a maximal (with respect to inclusion) $j$-connected subset of $X$.

Let $(k, \bar{k})$ be an ordered pair of adjacency relations. Throughout this article, it is assumed that $(k, \bar{k})$ belongs to $\{(1,2),(2,1)\}$. A $(k, \bar{k})$ binary digital picture (or, 
in short picture $)$ is a quadruple $(\mathcal{V}, k, \bar{k}, B)[15]$, where set $\mathcal{V}$ contains all points of the given grid, $B \subseteq \mathcal{V}$ denotes the set of black points, and each point in $\mathcal{V} \backslash B$ is said to be a white point. A black component or object is a $k$-component of $B$, while a white component is a $\bar{k}$-component of $\mathcal{V} \backslash B$.

Here it is assumed that a picture contains finitely many black points. Consequently there is a unique infinite white component, which is said to be the background. A finite white component is called a cavity in a picture.

A black point $p$ is an interior point if all points in $N_{\bar{k}}^{* \mathcal{V}}(p)$ are black. A black point $p$ is said to be a border point if $p$ is $\bar{k}$-adjacent to at least one white point (i.e., $N_{\bar{k}}^{* \mathcal{V}}(p) \backslash B \neq \emptyset$ ). A border-point $p$ is called an isolated point if all points in $N_{k}^{* \mathcal{V}}(p)$ are white (i.e., $\{p\}$ is a singleton object).

\subsection{Topology Preservation}

A reduction in a 2D picture is topology-preserving if each object in the input picture contains exactly one object in the output picture, and each white component in the output picture contains exactly one white component in the input picture [15]. In other words, a 2D reduction is topology-preserving if no object in the input picture is split (into two or more) or completely deleted, no cavity in the input picture is merged with the background or another cavity, and no cavity is created where there was none in the input picture [13].

Figure 3 depicts a counter-example.
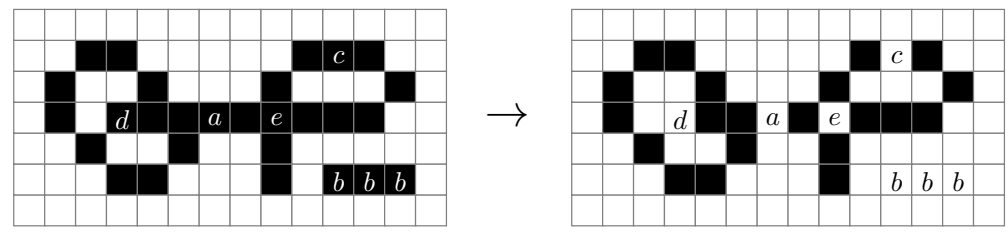

Figure 3: A reduction for a $(2,1)$ picture on $\mathbb{Z}^{2}$ that is not topology-preserving. Deletion of the point marked ' $a$ ' splits the larger object into two and the smaller object is completely deleted by deleting the points marked ' $b$ '; deletion of the point marked ' $c$ ' merges a cavity with the background; the remaining two cavities are merged with each other by deleting the point ' $d$ '; deletion of the point marked ' $e$ ' creates a brand new cavity.

\subsection{Simple Points}

A black point is said to be simple in a picture if its deletion is a topology-preserving reduction [13, 15]. In [15], Kong and Rosenfeld stated a characterization of simple points only on the square grid. Later Kardos and Palágyi stated a 'formal' and two kinds of 'easily visualized' characterizations of simple points in all the given five types of pictures on the regular $2 \mathrm{D}$ grids (i.e., two for $\mathcal{T}$, two for $\mathbb{Z}^{2}$, and one for 
$\mathcal{H})[9,10,12]$. The following theorem states our 'formal' necessary and sufficient condition:

Theorem 2.1. [12] Let $p$ be a black point in a picture $(\mathcal{V}, k, \bar{k}, B)$. Then $p$ is simple if and only if the following conditions hold:

1. $p$ is k-adjacent to exactly one $k$-component of $N_{2}^{* \mathcal{V}}(p) \cap B$.

2. $p$ is $\bar{k}$-adjacent to exactly one $\bar{k}$-component of $N_{2}^{\mathcal{V}}(p) \backslash B$.

Theorem 2.1 shows that simplicity of a point $p$ is a local property: it can be decided by examining the set $N_{2}^{* \mathcal{V}}(p)$ containing just 12,8 , and 6 points for $\mathcal{T}, \mathbb{Z}^{2}$, and $\mathcal{H}$, respectively. As a straightforward consequence of the above theorem we note that if a black point is an isolated or interior point then it is not simple (i.e., some border points may be simple). Another immediate consequence of Theorem 2.1 is the following duality theorem:

Theorem 2.2. A black point $p$ is simple in picture $(\mathcal{V}, k, \bar{k}, B)$ if and only if $p$ is simple in picture $(\mathcal{V}, \bar{k}, k,(\mathcal{V} \backslash B) \cup\{p\})$.

Figure 4a classifies the set of black points in a in a $(2,1)$ picture on $\mathbb{Z}^{2}$ into (non-simple) interior points, non-simple border points, and simple (border) points.

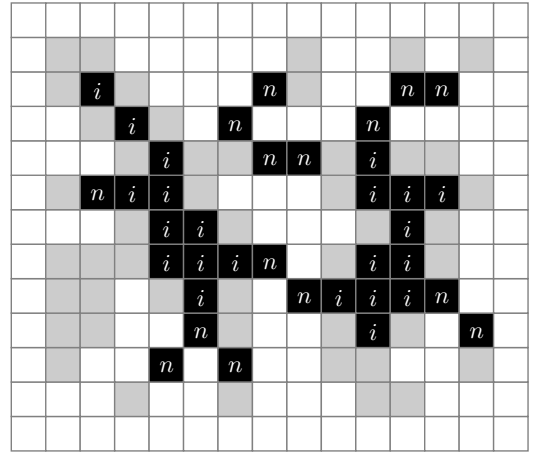

(a)

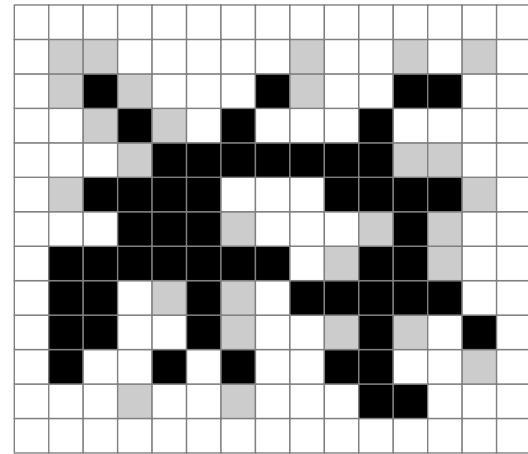

(b)

Figure 4: Classifying black points in a $(2,1)$ picture on $\mathbb{Z}^{2}$ (a). Notations: (nonsimple) interior points are marked ' $i$ '; non-simple border points are marked ' $n$ '; simple (border) points are depicted in gray. An example of $P$-simple sets in the same picture (b). Elements in that $P$-simple set are depicted in gray. Note that all possible $P$-simple sets are subsets of simple points.

\section{Sufficient Conditions for Topology-Preservation}

The deletion of a single point in a picture preserves the topology if and only if it is simple in that picture. However, reductions can delete one set of black points at a 
time. Hence we need a precise definition of what is meant by topology preservation when a number of points are deleted simultaneously.

Definition 3.1. [13, 17] Let $B$ be the set of black points in an arbitrary picture. $A$ set of $n$ points $Q \subset B$ is a simple set for $B$ if it is possible to arrange the elements of $Q$ in a sequence $\left\langle q_{1}, \ldots, q_{n}\right\rangle$ such that $q_{1}$ is a simple point for $B$ and each $q_{i}$ is simple after the set of points $\left\{q_{1}, \ldots, q_{i-1}\right\}$ is deleted $(i=2, \ldots, n)$. Such a sequence is called a simple sequence. (And let the empty set be called simple.)

Figure 5 gives examples of simple and non-simple sets of black points in a $(2,1)$ picture on the grid $\mathbb{Z}^{2}$.

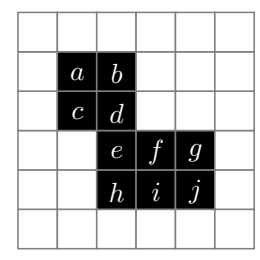

Figure 5: Examples of simple and non-simple sets in the picture $\left(\mathbb{Z}^{2}, 2,1,\{a, \ldots, j\}\right)$. The set of black points $\{a, b, c, d\}$ is simple as all the 12 sequences (of the possible 24 ones) $\langle a, b, c, d\rangle,\langle a, b, d, c\rangle,\langle a, c, b, d\rangle,\langle a, d, b, c\rangle$, $\langle b, a, c, d\rangle,\langle b, a, d, c\rangle,\langle b, c, a, d\rangle,\langle b, d, a, c\rangle,\langle c, a, b, d\rangle,\langle c, b, a, d\rangle,\langle d, a, b, c\rangle$, and $\langle d, b, a, c\rangle$ are simple. The set of black points $\{f, i\}$ is non-simple as both sequences $\langle f, i\rangle$ and $\langle i, f\rangle$ are non-simple. (Note that all black points are simple in this picture. Hence all the 10 singleton sets $\{a\}, \ldots,\{j\}$ are simple sets.)

There is general agreement that the concept of a simple set trivially implies a sufficient condition for topology-preserving reductions:

Criterion 3.1. [13, 17, 33] A reduction is topology-preserving if, for all possible pictures, it deletes only simple sets.

\section{$3.1 \quad P$-Simple Sets}

Bertrand introduced the notion of a $P$-simple set, whose simultaneous deletion preserves the topology:

Definition 3.2. [1] Let $B$ be the set of black points in an arbitrary picture. A set of black points $Q \subset B$ is a $P$-simple set for $B$ if for any point $q \in Q$ and any set of points $R \subseteq Q \backslash\{q\}, q$ is simple for $B \backslash R$. Each element of a $P$-simple set is called a $P$-simple point.

Figure $4 \mathrm{~b}$ shows an example of $P$-simple sets in a $(2,1)$ picture on $\mathbb{Z}^{2}$.

Theorem 3.1. [1] A reduction that deletes a subset composed solely of P-simple points is topology-preserving. 
Note that Bertrand and Couprie gave a local characterization of $P$-simple points in $(2,1)$ pictures on $\mathbb{Z}^{2}[3]$. Kardos and Palágyi presented both 'formal' characterization and 'easily visualized' sufficient and necessary conditions of $P$-simple points in all the five given types of pictures [11].

\subsection{Hereditarily Simple Sets}

Kong reported an alternative solution to the problem by introducing the notion of a hereditarily simple set, whose simultaneous deletion is proved to be topologypreserving [13].

Definition 3.3. [13] Let $B$ be the set of black points in an arbitrary picture. A set of points $Q \subset B$ is said to be hereditarily simple for $B$ if all subsets of $Q$ (including $Q$ itself) are simple sets in that picture.

Theorem 3.2. [13] A reduction that deletes only hereditarily simple sets is topology-preserving.

\subsection{Configuration-Based Condition}

Ronse [33] and later Kong [13] gave a sufficient condition for topology-preserving reductions acting on $(2,1)$ pictures on $\mathbb{Z}^{2}$. This condition concerns some configurations of deleted points, hence it is referred to as a configuration-based condition. Kardos and Palágyi formulated the following unified configuration-based sufficient condition:

Definition 3.4. [8] An object in a picture $(\mathcal{V}, 2,1, B)$ is small if it is composed of two or more mutually 2-adjacent points, and it is not formed by two 1-adjacent points.

Theorem 3.3. [8] For any picture $(\mathcal{V}, k, \bar{k}, B)$, a reduction is topology-preserving if all of the following conditions hold.

1. Only simple points for $B$ are deleted.

2. For any two $\bar{k}$-adjacent black points $p, q \in B$ that are deleted, $p$ is simple for $B \backslash\{q\}$.

3. If $(k, \bar{k})=(2,1)$, no small object is deleted completely.

\subsection{Point-Based Conditions}

Condition 2 of Theorem 3.3 takes pairs of $\bar{k}$-adjacent deleted points into consideration, and Condition 3 applies to small objects. Hence this theorem just provides a method of verifying that a formerly constructed reduction preserves the topology, rather than a methodology for constructing topology-preserving reductions. This is why point-based conditions were proposed that directly provide deletion rules 
of topology-preserving reductions, and allow us to construct topology-preserving thinning algorithms [21, 22].

Kardos and Palágyi proposed the following theorem that states the deletability of individual points:

Theorem 3.4. $[9,10,12]$ A reduction acting on $(k, \bar{k})$ pictures on $\mathcal{V}$ is topology preserving, if for any set of black points $B$ and for any point $p \in B$ that is deleted by that reduction, the following conditions hold:

1. Point $p$ is simple for $B$.

2. For any point $q \in N_{\bar{k}}^{* \mathcal{V}}(p) \cap B$ that is simple for $B$, point $p$ is simple for $B \backslash\{q\}$.

3. For the $(k, \bar{k})=(2,1)$ case, $p$ is not an element of a small object.

Conditions of Theorem 3.4 may be viewed as symmetric since elements in pairs of $\bar{k}$-adjacent points (see Condition 2) and points in small objects (see Condition 3) are not distinguished.

We examined some total orderings of elements in the given three regular planar grids. Now let us assume the addressing schemes depicted in Fig. 6, which define every point in $\mathbb{Z}^{2}$ and $\mathcal{H}$ by a pair of coordinates and the lexicographical order relation ' $\prec$ ' between two distinct points $p=\left(p_{x}, p_{y}\right)$ and $q=\left(q_{x}, q_{y}\right)$ is defined as follows: $p \prec q \Leftrightarrow\left(p_{y}<q_{y}\right) \vee\left(\left(p_{y}=q_{y}\right) \wedge\left(p_{x}<q_{x}\right)\right)$. Let $Q$ be a finite set of points. Then, point $p \in Q$ is said to be the smallest element of $Q$ if for any $q \in Q \backslash\{p\}, p \prec q$.
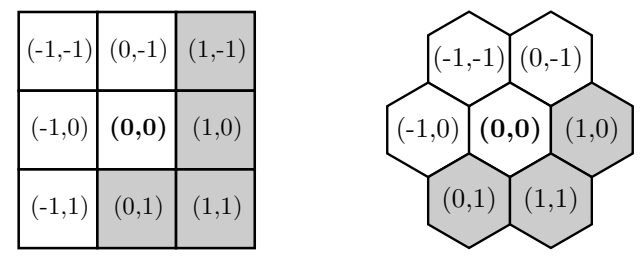

Figure 6: Feasible addressing schemes for the grids $\mathbb{Z}^{2}$ and $\mathcal{H}$. Each point $q$ in $N_{2}^{* \mathbb{Z}^{2}}(p)$ and $N_{2}^{* \mathcal{H}}(p)$ such that $p \prec q$ is depicted in gray, where $p$ is the central point with coordinates $(0,0)$.

With the help of the proposed ordering, Kardos and Palágyi gave the following asymmetric point-based condition for topology-preserving reductions:

Theorem 3.5. $[9,10,31]$ A reduction acting on $(k, \bar{k})$ pictures on $\mathcal{V}$ is topology preserving, if for any set of black points $B$ and for any point $p \in B$ that is deleted by that reduction the following conditions hold:

1. Point $p$ is simple for $B$. 
2. For any point $q \in N_{\bar{k}}^{* \mathcal{V}}(p) \cap B$ that is simple for $B$ and $p \prec q$, point $p$ is simple for $B \backslash\{q\}$.

3. For the $(k, \bar{k})=(2,1)$ case, $p$ is not the smallest element of a small object.

Note that Kardos and Palágyi marked the smaller point in the possible pairs of $\bar{k}$-adjacent points, and the smallest point in the possible small objects on $\mathcal{T}[10]$. Therefore relation ' $\prec$ ' on the triangular grid has also been defined.

Our symmetric and asymmetric point-based sufficient conditions (see theorems 3.4 and 3.5) allow us to derive the following reductions:

Definition 3.5. Let $\mathrm{R}_{\text {symm }}^{\mathcal{V},(k, \bar{k})}$ be the reduction acting on $(k, \bar{k})$ pictures on $\mathcal{V}$ that deletes all points satisfying all conditions of Theorem 3.4.

Definition 3.6. Let $\mathrm{R}_{a s y m m}^{\mathcal{V},(k, \bar{k})}$ be the reduction acting on $(k, \bar{k})$ pictures on $\mathcal{V}$ that deletes all points satisfying all conditions of Theorem 3.5.

Note that all the five pairs of the derived reductions are evidently topologypreserving. Figure 7 gives an example of the pair of reductions acting on the hexagonal grid.
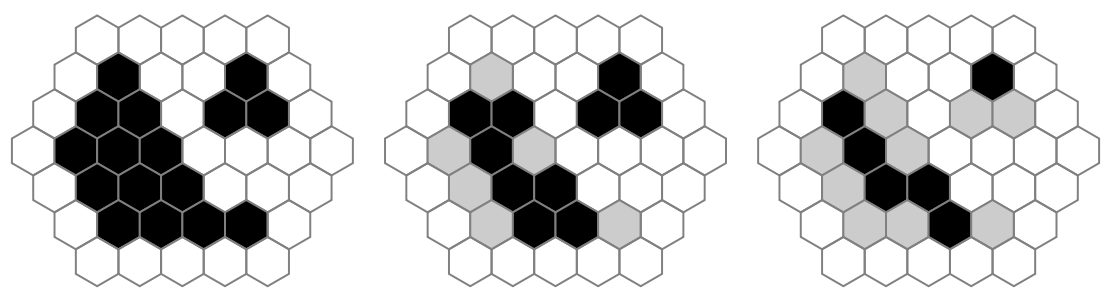

Figure 7: The original picture on the hexagonal grid $\mathcal{H}$ (left) and the results produced by the two point-based reductions $\mathrm{R}_{\text {symm }}^{\mathcal{H},(1,2)}=\mathrm{R}_{\text {symm }}^{\mathcal{H},(2,1)}$ (middle) and $\mathrm{R}_{\text {asymm }}^{\mathcal{H},(1,2)}=\mathrm{R}_{\text {asymm }}^{\mathcal{H},(2,1)}$ (right). Deleted pixels are depicted in gray.

\subsection{General-Simple Deletion Rules}

Each sufficient condition for topology-preserving reductions reported here checks some configurations of deleted points or individual deleted points. The author proposed a novel condition that considers the deletion rules of reductions $[23,25,27]$ that specify the points to be deleted.

Parallel reductions can change a set of black points simultaneously, while sequential reductions traverse the black points of a picture, and focus on the actually visited single point for possible deletion. These two absolutely dissimilar strategies are illustrated in algorithms 1 and 2.

Thinning algorithms generally classify the set of black points in input pictures into two (disjoint) subsets. That is, the deletion rule associated with a phase 

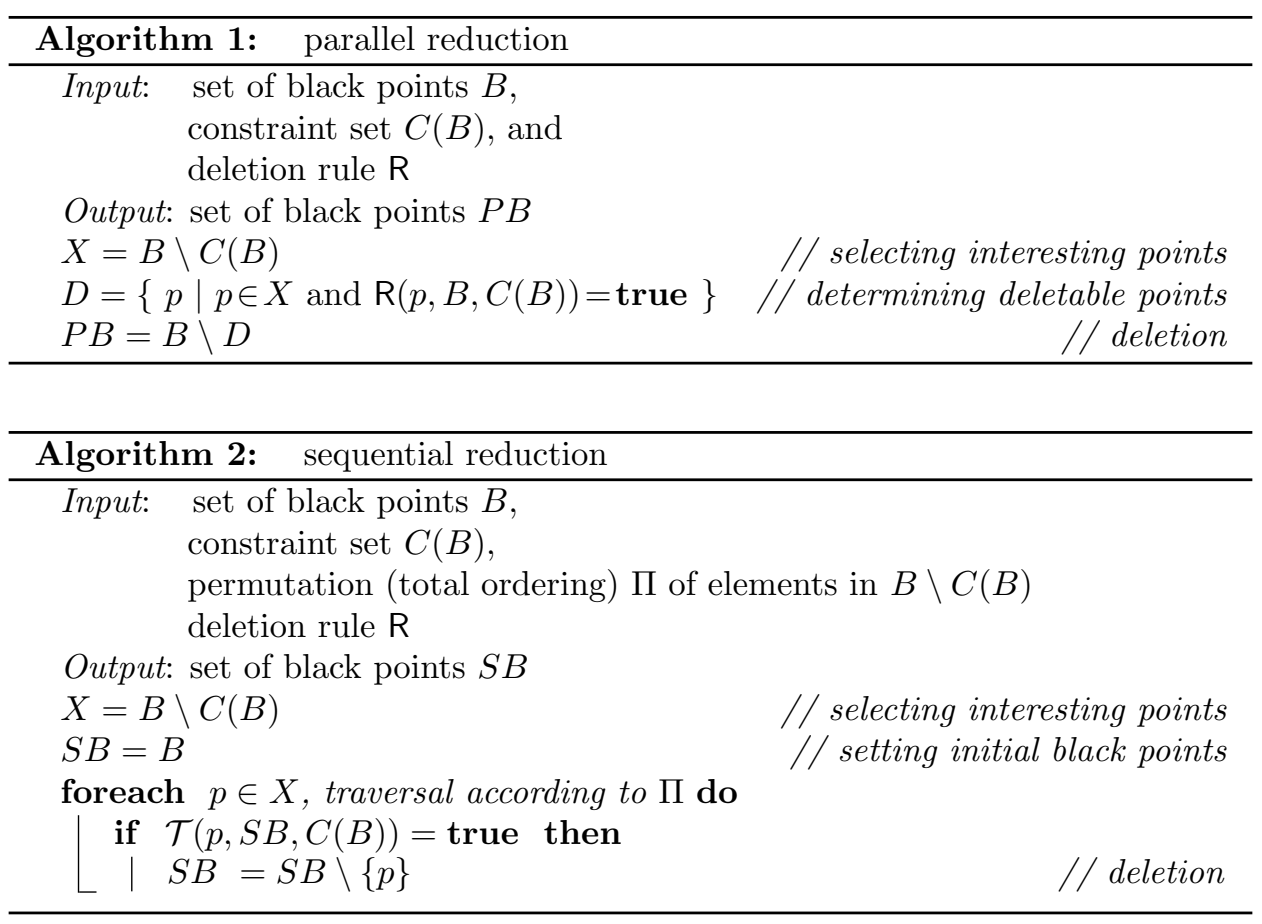

of an algorithm is evaluated for the elements of its set of interesting points, and black points in its constraint set are not taken into consideration. This is why algorithms 1 and 2 treat a constraint set $C(B) \subset B$ (as an input parameter) and its complementary $X=B \backslash C(B)$ as a set of interesting points.

An interesting point $p \in X$ is deletable by the deletion rule $\mathrm{R}$, if $\mathrm{R}(p, Y, C(B))=$ true, where $Y$ denotes the set of black points in the (actual) picture, i.e., $Y=S B \subseteq$ $B$ in sequential reductions (see Algorithm 2), and $Y=B$ in the parallel case (see Algorithm 1). Therefore, in the parallel case the initial picture is considered when the deletion rule is evaluated. In contrast, the picture is dynamically altered when a sequential reduction is performed. We should add that elements of the constraint set $C(B)$ are omitted when the deletion rule $\mathrm{R}$ is evaluated. For practical purposes, we will deal with finite pictures (i.e., $B$ contains finitely many points).

The sequential approach suffers from the drawback that different visiting orders of interesting points may yield different results. A deletion rule $\mathrm{R}$ is said to be orderindependent if the result of Algorithm 2 is uniquely specified by R (i.e., the result of Algorithm 2 does not depend on the order $\Pi$ in which the points are selected by the foreach loop) [7, 23, 32].

Two reductions are called equivalent if they produce the same result for each input picture. A deletion rule is said to be equivalent if it yields a pair of equivalent parallel and sequential reductions.

The support of a deletion rule $\mathrm{R}$ applied at a point is a minimal set of points 
whose values determine whether the investigated points are deleted by $\mathrm{R}$ from a picture. Note that thinning and shrinking algorithms use local supports with 'small' diameters. Let us denote the support of the deletion rule $\mathrm{R}$ with respect to a point $p$ by $\mathbf{S}_{\mathrm{R}}(p)$. (Generally $N_{2}^{* \mathcal{V}}(p) \subseteq \mathbf{S}_{\mathrm{R}}(p) \subseteq \bigcup_{q \in N_{2}^{\mathcal{V}}(p)} N_{2}^{\mathcal{V}}(q) \backslash\{p\}$.) It is easy to see that $\mathrm{R}(p, Y, C(B))=\mathrm{R}\left(p, Y \cap \mathbf{S}_{\mathrm{R}}(p), C(B) \cap \mathbf{S}_{\mathrm{R}}(p)\right)$.

The author introduced two special classes of deletion rules. These are:

Definition 3.7. [25] Let $\mathrm{R}$ be a deletion rule, let $B$ be a set of black points in a picture, let $p \in B \backslash C(B)$ be an interesting point with respect to the constraint set $C(B) \subset B$, and let us assume that $\mathrm{R}(p, B, C(B))=$ true (i.e., $p$ can be deleted by $\mathrm{R})$. Then $R$ is general if $\mathrm{R}(q, B, C(B))=\mathrm{R}(q, B \backslash\{p\}, C(B))$ for any point $q \in B \backslash C(B)$.

In other words, a deletion rule is general if the deletability of any point does not depend on the 'color' of any deletable point. It is obvious that a method of verifying that a deletion rule $\mathrm{R}$ is general may ignore each point $q \notin \mathbf{S}_{\mathrm{R}}(p)$.

Definition 3.8. [25] A deletion rule is general-simple if it is general, and it deletes only simple points.

The following theorems summarize the author's most important results concerning general and general-simple deletion rules:

Theorem 3.6. [25] A deletion rule $\mathrm{R}$ is order-independent if and only if $\mathrm{R}$ is general.

Theorem 3.7. [25] A deletion rule $\mathrm{R}$ is equivalent if $\mathrm{R}$ is general.

Theorem 3.8. [25] A (sequential or parallel) reduction is topology-preserving if its deletion rule is general-simple.

Theorem 3.8 is an exceptional, sufficient condition for topology-preserving reductions. In addition, with the help of general-simple deletion rules some sequential thinning algorithms can be directly implemented for parallel computers, and conversely, some parallel algorithms can be readily implemented on conventional sequential computers.

In [24], the author proved that the deletion rule of the 2D fully parallel thinning algorithm proposed by Manzanera et al. [18] is general-simple, and Palágyi, Németh, and Kardos gave a pair of equivalent 2D sequential and parallel subiterationbased thinning algorithms [28]. Palágyi, Németh, and Kardos proposed four pairs of equivalent sequential and parallel subiteration-based 3D surface-thinning algorithms [26], and Palágyi and Németh gave a pair of equivalent sequential and fully parallel 3D surface-thinning algorithms [30].

\section{Relationships}

Next, the relationships among the given five types of sufficient conditions are presented. 


\subsection{Deletion of Hereditarily Simple Sets and Deletion of $P$ - Simple Sets}

In [14], Kong and Gau proved that the two kinds of sufficient conditions for topology-preserving reductions based on $P$-simple sets (i.e., Theorem 3.1) and hereditarily simple sets (i.e., Theorem 3.2) are equivalent. We will state this as a theorem:

Theorem 4.1. [14] A set of black points in a picture is hereditarily simple if and only if it is a P-simple set in that picture.

\subsection{Configuration-Based and Point-Based Sufficient Condi- tions}

Let us now state the relationship between the point-based and the configurationbased conditions:

Theorem 4.2. If a reduction satisfies a point-based condition (see theorems 3.4 or 3.5), it satisfies the configuration-based condition (see Theorem 3.3) as well.

Proof. It can readily be seen that if a parallel reduction satisfies Condition $i$ of Theorem 3.4 (i.e., the symmetric point-based result), Condition $i$ of Theorem 3.3 (i.e., the configuration-based result) holds for each $i \in\{1,2,3\}$.

Similarly, it is clear that if a parallel reduction satisfies Condition $i$ of Theorem 3.5 (i.e., the asymmetric point-based result), Condition $i$ of Theorem 3.3 (i.e., the configuration-based result) holds for each $i \in\{1,2,3\}$.

\subsection{Configuration-Based Sufficient Conditions and Deletion of P-Simple Sets}

Palágyi and Kardos proved the following theorem:

Theorem 4.3. [31] If a reduction acting on $(k, \bar{k})$ pictures on $\mathcal{V}$ deletes only $P$ simple sets, all conditions of Theorem 3.3 (i.e., the configuration-based result) are satisfied.

We can also prove the following theorem as well:

Theorem 4.4. [31] If a reduction acting on $(k, \bar{k})$ pictures on $\mathcal{V}$ satisfies all conditions of Theorem 3.3, it deletes only P-simple sets.

In [31], we reported the proof of Theorem 4.4 for $(1,2)$ pictures on $\mathbb{Z}^{2}$. Here, it is carried out for the hexagonal case.

By Theorem 2.1, it can readily be seen that black simple points in $(1,2)=(2,1)$ pictures on $\mathcal{H}$ are characterized by the matching templates depicted in Fig. 8.

Since the simplicity of a point is a local property by Theorem 2.1 , the following proposition holds: 


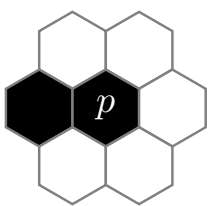

(a)

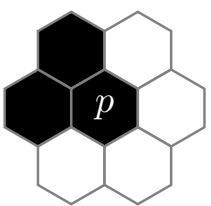

(b)

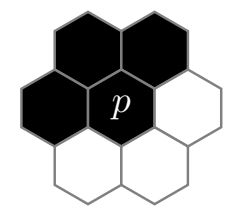

(c)

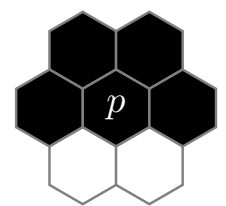

(d)

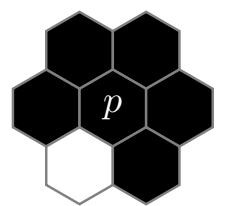

(e)

Figure 8: The five base matching templates for characterizing a black simple point $p$ in $(1,2)=(2,1)$ pictures on $\mathcal{H}$. Note that all the rotated and reflected versions of the base matching templates also match simple points.

Proposition 4.1. Let $Q \subset B$ be a set of points in a picture $(\mathcal{V}, k, \bar{k}, B)$. A point $q \in Q$ is a P-simple point for $Q$ if for any set of points $R \subseteq N_{2}^{* \mathcal{V}}(q) \cap Q, q$ is simple for $B \backslash R$.

Theorem 4.5. If a parallel reduction obeys all the conditions of Theorem 3.3 (i.e., the configuration-based result), and it deletes the set of points $Q \subset B$ from picture $(\mathcal{H}, 1,2, B)=(\mathcal{H}, 2,1, B)$, then $Q$ is a P-simple set.

Proof. Let $p \in Q$. Since Condition 1 of Theorem 3.3 holds, $p$ is simple for $B$. Without loss of generality, we will just consider the five base matching templates shown in Fig. 8.

By Proposition 4.1, the following cases are to be investigated with the help of the configurations shown in Fig. 9:

(a) If $p$ is matched by the template in Fig. 8a, then consider the configuration in Fig. 9a. In this case, only the black point $q$ need be examined. Since $p$ is a non-simple (isolated black) point in $B \backslash\{q\}$, by Condition 2 of Theorem 3.3, $q \notin Q$.

(b) If $p$ is matched by the template in Fig. $8 \mathrm{~b}$, then consider the configuration in Fig. 9b. Let us investigate the two black points $q$ and $r$.

- Assume that $q \in Q$ and $r \notin Q$. Since $p$ is matched by the template in Fig. 8a in $B \backslash\{q\}, p$ remains simple after the deletion of $q$.

- Assume that $r \in Q$ and $q \notin Q$. Since $p$ is matched by the template in Fig. 8a in $B \backslash\{r\}, p$ remains simple after the deletion of $r$.

- Assume that $q \in Q$ and $r \in Q$. Since $q$ is a simple point, and it remains simple after the deletion of $r$, by Condition 2 of Theorem 2.1, all points in $\{c, d, e\}$ are white. Since $r$ is a simple point, and it remains simple after the deletion of $q$, by Condition 2 of Theorem 2.1, all points in $\{a, b, c\}$ are white. Since $\{p, q, r\}$ is a small object, by Condition 3 of Theorem 3.3, we arrive at a contradiction.

(c) If $p$ is matched by the template in Fig. 8c, then consider the configuration in Fig. 9c. It can readily be seen that $p$ is not simple for $B \backslash\{r\}$. Hence, by 
Condition 2 of Theorem 3.3, $r \notin Q$. Now let us examine the remaining two black points $q$ and $s$.

- Assume that $q \in Q$ and $s \notin Q$. Since $p$ is matched by the template in Fig. $8 \mathrm{~b}$ in $B \backslash\{q\}, p$ remains simple after the deletion of $q$.

- Assume that $s \in Q$ and $q \notin Q$. Since $p$ is matched by the template in Fig. 8b in $B \backslash\{s\}, p$ remains simple after the deletion of $s$.

- Assume that $q \in Q$ and $s \in Q$. Since $p$ is matched by the template in Fig. 8a in $B \backslash\{q, s\}, p$ remains simple after the deletion of $\{q, s\}$.

(d) If $p$ is matched by the template in Fig. 8d, then consider the configuration in Fig. 9d. It can readily be seen that $p$ is not simple for $B \backslash\{r\}$ and $B \backslash\{s\}$. Hence, by Condition 2 of Theorem 3.3, $r \notin Q$ and $s \notin Q$. Now let us examine the remaining two black points $q$ and $t$.

- Assume that $q \in Q$ and $t \notin Q$. Since $p$ is matched by the template in Fig. 8c in $B \backslash\{q\}, p$ remains simple after the deletion of $q$.

- Assume that $t \in Q$ and $q \notin Q$. Since $p$ is matched by the template in Fig. $8 \mathrm{c}$ in $B \backslash\{t\}, p$ remains simple after the deletion of $t$.

- Assume that $q \in Q$ and $t \in Q$. Since $p$ is matched by the template in Fig. 8b in $B \backslash\{q, t\}, p$ remains simple after the deletion of $\{q, t\}$.

(e) If $p$ is matched by the template in Fig. 8e, then consider the configuration in Fig. 9e. It can readily be seen that $p$ is not simple for $B \backslash\{r\}, B \backslash\{s\}$, and

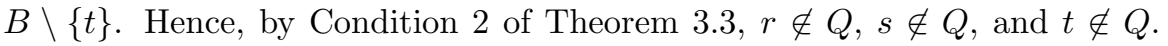
Now let us examine the remaining two black points $q$ and $u$.

- Assume that $q \in Q$ and $u \notin Q$. Since $p$ is matched by the template in Fig. $8 \mathrm{~d}$ in $B \backslash\{q\}, p$ remains simple after the deletion of $q$.

- Assume that $u \in Q$ and $q \notin Q$. Since $p$ is matched by the template in Fig. 8d in $B \backslash\{u\}, p$ remains simple after the deletion of $u$.

- Assume that $q \in Q$ and $u \in Q$. Since $p$ is matched by the template in Fig. 8c in $B \backslash\{q, u\}, p$ remains simple after the deletion of $\{q, u\}$.

Since $p$ remains simple after the deletion of each subset of $Q, p$ is a $P$-simple point for $Q$.

In [2], Bertrand proposed a two-step (topology-preserving) thinning scheme that is based on $P$-simple points. One phase/reduction of the iterative thinning process is performed as follows:

1. A set of points $Q \subset B$ is (somehow) chosen and labeled.

2. All $P$-simple points in $Q$ are deleted (simultaneously). 


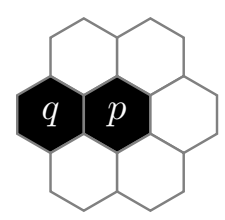

(a)

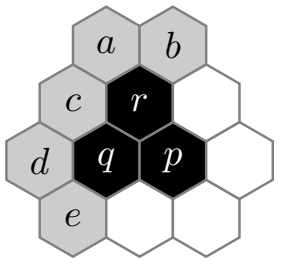

(b)

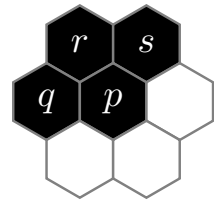

(c)

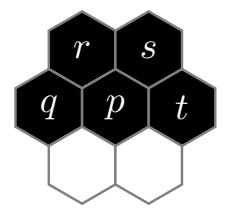

(d)

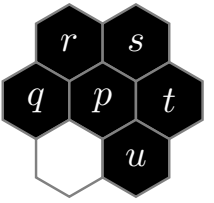

(e)

Figure 9: Configurations associated with Theorem 4.5 concerning $(1,2)=(2,1)$ pictures on $\mathcal{H}$.

Note that Step 2 concerns tricolor pictures (say: the value ' 0 ' corresponds to white points, the value ' 1 ' is assigned to (black) points in $B \backslash Q$, and value ' 2 ' corresponds to (black) points in $Q$ ). Hence this two-step scheme is both space- and time-consuming.

Theorems 4.2 and 4.4 provide a single-step thinning scheme that deletes $P$ simple points as well. The deletion rule of a reduction of the iterative thinning process can be directly constructed by combining the reduction $\mathrm{R}_{s y m m}^{\mathcal{V},(k, \bar{k})}$ (see Definition 3.5) or $\mathrm{R}_{a s y m m}^{\mathcal{V},(k, \bar{k})}$ (see Definition 3.6) with different thinning strategies (i.e., fully parallel, subiteration-based, and subfield-based [5]) and various geometric constraints (say endpoints [5]). The generated deletion rule is a common Boolean function that is to be evaluated for the neighborhood of the points in question in binary (two-level) pictures. As this Boolean function can be stored in a pre-calculated look-up-table, the proposed single-step scheme can be implemented efficiently.

\subsection{Deletion of P-Simple Sets and General-Simple Deletion Rules}

Let us consider some important properties of $P$-simple sets and general-simple deletion rules:

Proposition 4.2. Let $B$ be the set of black points in an arbitrary picture, and let $p$ be an arbitrary point in a $P$-simple set $Q$ for $B$. Then $p$ is simple for $B$.

Proof. Since $\emptyset \subset Q$, by Definition 3.2, $p$ is simple for $B \backslash \emptyset=B$.

Proposition 4.3. Let $B$ be the set of black points in an arbitrary picture, and let $Q$ be a $P$-simple set for $B$. Then $R$ is a $P$-simple set for $B \backslash(Q \backslash R)$ for any $R \subset Q$.

Proof. Consider a point $r \in R$ and a set of points $T \subseteq R \backslash\{r\}$. Since $r \in Q$, $T \cup(Q \backslash R) \subseteq Q \backslash\{r\}$, and $Q$ is a $P$-simple set for $B, r$ is simple for $B \backslash(T \cup(Q \backslash R))=$ $(B \backslash(Q \backslash R)) \backslash T$. Hence, $R$ is a $P$-simple set for $B \backslash(Q \backslash R)$. 
Proposition 4.4. Any set of points $Q \subset B$ is a $P$-simple set for $B$ if and only if all possible permutations of $Q$ form simple sequences.

Proof. First, let $Q \subset B$ be a $P$-simple set of $n$ points, and consider the permutation/sequence of its elements $\left\langle q_{1}, \ldots, q_{n}\right\rangle$. Let us investigate the prefixes $\left\langle q_{1}\right\rangle$, $\left\langle q_{1}, q_{2}\right\rangle, \ldots\left\langle q_{1}, \ldots, q_{n-1}\right\rangle$ of that sequence. By Proposition 4.2, point $q_{1}$ is simple. Since $Q$ is a $P$-simple set, and $\left\{q_{1}, \ldots, q_{m-1}\right\} \subseteq Q \backslash\left\{q_{m}\right\}$ for each $m=2, \ldots, n$, point $q_{m}$ is simple for $B \backslash\left\{q_{1}, \ldots, q_{m-1}\right\}$. Hence, $\left\langle q_{1}, \ldots, q_{n}\right\rangle$ is a simple sequence.

Then let us assume that all possible permutations of a set $Q \subset B$ form simple sequences. Consider any point $q \in Q$ and any set of $n>0$ points $R=\left\{r_{1}, \ldots, r_{n}\right\}$ such that $R \subseteq Q \backslash\{q\}$. Since all prefixes of a simple sequence form simple sequences, $\left\langle r_{1}, \ldots, r_{n}, p\right\rangle$ is also a simple sequence. Consequently, $q$ is a simple point for $B \backslash R$. Thus $Q$ is a $P$-simple set.

Proposition 4.5. If a deletion rule is general-simple, it is order-independent.

Proof. By Definition 3.8, each general-simple deletion rule is general. Since, by Theorem 3.6, general deletion rules are order-independent, general-simple deletion rules are also order-independent.

Proposition 4.6. A deletion rule is equivalent if it is general-simple.

Proof. It is actually a direct consequence of Definition 3.8 and Theorem 3.7.

Proposition 4.7. All permutations of the elements in the set of points deleted by a (sequential or parallel) reduction with a general-simple deletion rule form simple sequences.

Proof. Let $\mathrm{R}$ be a general-simple deletion rule, and consider the sequential reduction (see Algorithm 2) with R. By Theorem 3.6, Theorem 3.8, and Proposition 4.5, the sequential reduction with $\mathrm{R}$ is order-independent and topology-preserving. Consequently, the result of Algorithm 2 does not depend on the order $\Pi$ in which the points in the set of interesting points $X$ are selected in the foreach loop. Rule $\mathrm{R}$ is equivalent, by Proposition 4.6, hence the parallel reduction (see Algorithm 1) with $\mathrm{R}$ deletes the same set of $n$ points $D \subseteq X$. Consider the arbitrary sequence of elements in the set of $n$ points $\left\langle d_{1}, d_{2}, \ldots, d_{n}\right\rangle$. Since $\mathrm{R}$ deletes only simple points, $d_{1}$ is simple, and $d_{m}$ is simple after the deletion of $\left\{d_{1}, \ldots, q_{m-1}\right\}(m=2, \ldots, n)$. Thus $\left\langle d_{1}, d_{2}, \ldots, d_{n}\right\rangle$ is a simple sequence.

We can state the following theorem as an immediate consequence of propositions 4.4 and 4.7 .

Theorem 4.6. Reductions with general-simple deletion rules delete P-simple sets.

Now we show that the contrary statement does not hold.

Theorem 4.7. The deletion rule of a reduction that deletes only P-simple sets may not be general-simple. 

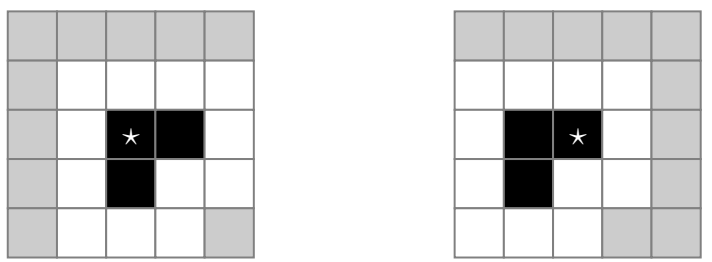

Figure 10: Matching templates associated with $\mathrm{R}$ working on $(2,1)$ pictures on $\mathbb{Z}^{2}$. The new value of a black point depends on its $5 \times 5$ neighborhood. A point is deletable by $\mathrm{R}$ if at least one template matches it. Notations: the position indicated by ' $\star$ ' is the center of the template; each black element matches a black point; each white element matches a white point; each gray element matches either a black or a white point.

Proof. Consider the plain deletion rule $\mathrm{R}$ that is given by two matching templates (see Fig. 10).

It can readily be seen that the parallel reduction with $\mathrm{R}$ obeys all the conditions of Theorem 3.3 (i.e., the configuration-based condition for topology-preserving reductions). By Theorem 4.4, this parallel reduction deletes only $P$-simple sets.

It is obvious that the parallel reduction with $\mathrm{R}$ deletes both upper points of a kind of small objects (see Definition 3.4) composed of three points (and nothing else). In contrast, the sequential reduction with $\mathrm{R}$ can delete just one upper point that is visited first. Hence, this sequential reduction is not order-independent. Thus $\mathrm{R}$ is not general by Theorem 2.1, and it is not general-simple by Definition 3.8.

Note that the author constructed a special deletion rule that deletes only $P$ simple points, and he proved that it is general-simple [29].

Lastly, we state the following theorem:

Theorem 4.8. For each $P$-simple set $Q$ in a picture, there is a general-simple deletion rule that deletes $Q$ from this picture.

Proof. Let $Q \subset B$ be a $P$-simple set for $B$. Consider the parallel and sequential reductions (see algorithms 1 and 2 ) with the following deletion rule:

$$
\mathrm{R}(q, S B, B \backslash Q)= \begin{cases}\text { true } & \text { if } q \text { is a } P \text {-simple point } \\ \text { false } & \text { otherwise }\end{cases}
$$

where $S B \subseteq B$ is the set of black points in the actual picture (that is initially equal to $B$ ), the constraint set $C(B)$ is $B \backslash Q$, and the set of interesting points $X$ is $Q$.

It is obvious that the parallel reduction with $\mathrm{R}$ deletes the $P$-simple set $Q$ (and nothing else).

To prove this theorem, it is necessary to show that $\mathrm{R}$ is general-simple. By Proposition $4.2, \mathrm{R}$ deletes only simple points. Hence the only thing we need to verify is that deletion rule $R$ is general. 
Consider a set of points $D \subseteq Q$, and two points $p, q \in Q \backslash D$, and let us assume that $S B=B \backslash D$. Since $Q \subset B$ is a $P$-simple set for $B$, by Proposition 4.3, both points $p$ and $q$ are $P$-simple for $S B$, and $q$ are $P$-simple for $S B \backslash\{p\}$. Consequently, $\mathrm{R}(p, S B, B \backslash Q)=$ true and $\mathrm{R}(q, S B, B \backslash Q)=\mathrm{R}(q, S B \backslash\{p\}, B \backslash Q)$. Thus $\mathrm{R}$ is general.

\subsection{Summary of Relationships}

Here, we summarize the relationships among the five types of sufficient conditions for topology-preserving reductions with the help of Fig. 11. Note that three of them (namely: deletion of $P$-simple sets, deletion of hereditarily simple sets, and general-simple deletion rules) are absolutely universal, and the relationships among them are valid for arbitrary pictures.

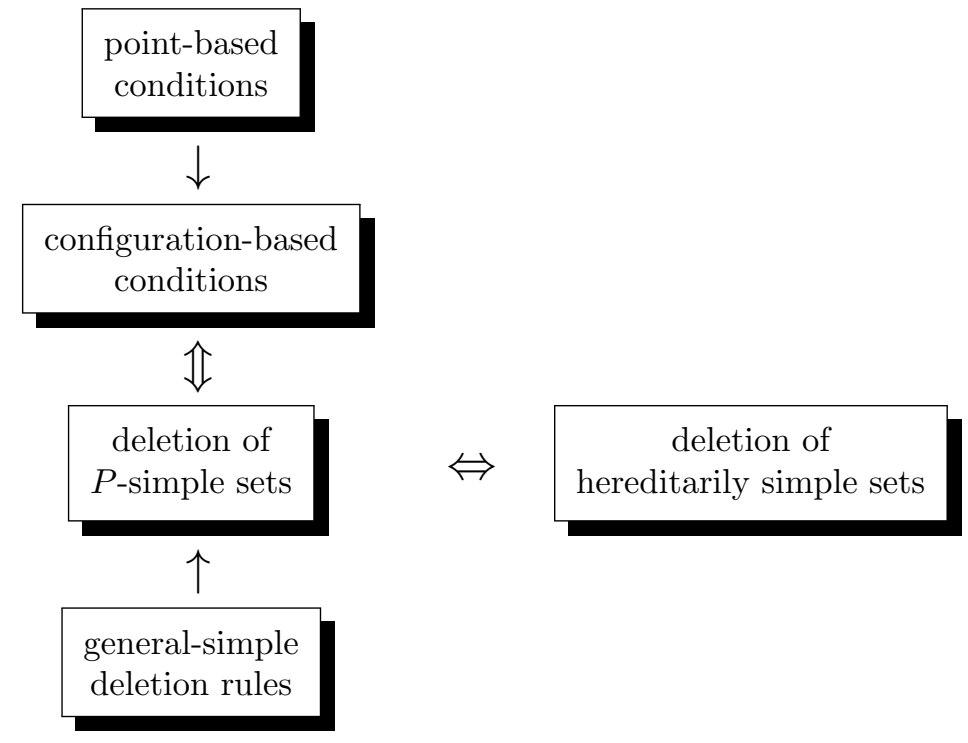

Figure 11: How the five kinds of sufficient conditions for topology-preserving reductions are related.

The linkage between $P$-simple sets and hereditarily simple sets was established by Kong and Gau [14], and the remaining relationships were discovered by Palágyi and Kardos.

\section{Conclusions}

In this paper, five types of sufficient conditions for topology-preserving reductions acting on the three possible regular planar grids are reported, and relationships among these conditions were presented. These conditions are based on configurations, individual deletable points, $P$-simple sets, hereditarily simple sets, and 
general-simple deletion rules. The given sufficient conditions are absolutely not autotelic, they provide methods of verifying that a reduction preserves the topology, allow us to generate topology-preserving reductions, and they provide computationally efficient thinning algorithms.

\section{References}

[1] Bertrand, G. On P-simple points. Compte Rendu de l'Académie des Sciences de Paris, Série Math., 321:1077-1084, 1995.

[2] Bertrand, G. P-simple points: A solution for parallel thinning. In: Proc. 5th Int. Conference on Discrete Geometry for Computer Imagery, DGCI 2005, pages 233-242, 1995.

[3] Bertrand, G., Couprie, M. Two-dimensional parallel thinning algorithms based on critical kernels. Journal of Mathematical Imaging and Vision, 31:35-56, 2008.

[4] Brimkov, V.E., Barneva, R.P. Analytical honeycomb geometry for raster and volume graphics. The Computer Journal, 48:180-199, 2005.

[5] Hall, R.W. Parallel connectivity-preserving thinning algorithms. In Kong, T.Y., Rosenfeld, A., editors, Topological algorithms for digital image processing, pages 145-179, Amsterdam, 1996. Elsevier Science B.V.

[6] Hall, R.W., Kong, T.Y., Rosenfeld, A. Shrinking binary images. In Kong, T.Y., Rosenfeld, A., editors, Topological algorithms for digital image processing, pages 31-98, Amsterdam, 1996. Elsevier Science B.V.

[7] Kardos, P., Palágyi, K. Order-independent sequential thinning in arbitrary dimensions. In: Proc. Int. Conference on Signal and Image Processing and Applications, SIPA 2011, pages 129-134, 2011.

[8] Kardos, P., Palágyi, K. On topology preservation in triangular, square, and hexagonal grids. In: Proc. 8th Int. Symposium on Image and Signal Processing and Analysis, IEEE/EURASIP, ISPA 2013, pages 782-787, 2013.

[9] Kardos, P., Palágyi, K. Topology-preserving hexagonal thinning. Int. Journal of Computer Mathematics, 90:1607-1617, 2013.

[10] Kardos, P., Palágyi, K. Topology preservation on the triangular grid. Annals of Mathematics and Artificial Intelligence, 75:53-68, 2015.

[11] Kardos, P., Palágyi, K. Unified characterization of $P$-simple points in triangular, square, and hexagonal grids. In: Proc. Int. Symposium on Computational Modeling of Objects Presented in Images: Fundamentals, Methods, and Applications, CompIMAGE'16, LNCS 10149, pages 79-88, 2016, Springer. 
[12] Kardos, P., Palágyi, K. On topology preservation of mixed operators in triangular, square, and hexagonal grids. Discrete Applied Mathematics, 216: 441-448, 2017.

[13] Kong, T.Y. On topology preservation in 2-D and 3-D thinning. Int. Journal of Pattern Recognition and Artificial Intelligence, 9:813-844, 1995.

[14] Kong, T.Y., Gau, C-J. Minimal non-simple sets in 4-dimensional binary images with (8,80)-adjacency. In: Combinatorial Image Analysis, IWCIA 2004, LNCS 3322, pages 318-333, 2004, Springer.

[15] Kong, T.Y., Rosenfeld, A. Digital topology: Introduction and survey. Computer Vision, Graphics, and Image Processing, 48:357-393, 1989.

[16] Kovalevsky, V.A. Geometry of locally finite spaces. Publishing House, 2008.

[17] Ma, C.M. On topology preservation in 3D thinning. CVGIP: Image Understanding, 59:328-339, 1994.

[18] Manzanera, A., Bernard, T.M., Pretêux, F., Longuet, B. n-dimensional skeletonization: a unified mathematical framework. Journal of Electronic Imaging, $11: 25-37,2002$.

[19] Marchand-Maillet, S., Sharaiha, Y.M. Binary digital image processing - A discrete approach. Academic Press, 2000.

[20] Middleton, L., Sivaswamy, J. Hexagonal image processing: A practical approach, Advances in Pattern Recognition. Springer-Verlag, 2005.

[21] Németh, G., Kardos, P., Palágyi, K. 2D parallel thinning and shrinking based on sufficient conditions for topology preservation. Acta Cybernetica, 20:125$144,2011$.

[22] Németh, G., Palágyi, K. Topology preserving parallel thinning algorithms. International Journal of Imaging Systems and Technology 21:37-44, 2011.

[23] Palágyi, K. Deletion rules for equivalent sequential and parallel reductions. In: Proc. 18th Iberoamerican Congress on Pattern Recognition, Part I, CIARP 2013, LNCS 8285, pages 17-24, 2013, Springer.

[24] Palágyi, K. Equivalent 2D sequential and parallel thinning algorithms. In: Proc. 16th Int. Workshop on Combinatorial Image Analysis, IWCIA 2014, LNCS 8466, pages 91-100, 2014, Springer.

[25] Palágyi, K. Equivalent sequential and parallel reductions in arbitrary binary pictures. Int. J. Pattern Recognition and Artificial Intelligence, 28:1460009-11460009-16, 2014. 
[26] Palágyi, K., Németh, G., Kardos, P. Equivalent sequential and parallel subiteration-based surface-thinning algorithms. In: Proc. 17th Int. Workshop on Combinatorial Image Analysis, IWCIA 2015, LNCS 9448, pages 31-45, 2015, Springer.

[27] Palágyi, K. Topology-preserving general operators in arbitrary binary pictures. In: Proc. 19th Iberoamerican Congress on Pattern Recognition, CIARP 2014, LNCS 8827, pages 22-29, 2014, Springer.

[28] Palágyi, K., Németh, G., Kardos, P. Topology-preserving equivalent parallel and sequential 4-subiteration 2D thinning algorithms. In: Proc. 9th Int. Symposium on Image and Signal Processing and Analysis, IEEE/EURASIP, ISPA 2015, pages 306-311, 2015.

[29] Palágyi, K. P-simple points and general-simple deletion rules. In: Proc. 19th IAPR Int. Conference on Discrete Geometry for Computer Imagery, DGCI 2016, LNCS 9647, pages 143-153, 2016, Springer.

[30] Palágyi, K., Németh, G. A pair of equivalent sequential and fully parallel 3D surface-thinning algorithms. Discrete Applied Mathematics, 216:348-361, 2017.

[31] Palágyi, K., Kardos, P. A single-step 2D thinning scheme with deletion of $P$ simple points. In: Proc. 22nd Iberoamerican Congress on Pattern Recognition, CIARP 2017, LNCS 1065\%, in press.

[32] Ranwez, V., Soille, P. Order independent homotopic thinning for binary and grey tone anchored skeletons. Pattern Recognition Letters, 23:687-702, 2002.

[33] Ronse, C. Minimal test patterns for connectivity preservation in parallel thinning algorithms for binary digital images. Discrete Applied Mathematics, 21:67-79, 1988 . 